\title{
Effect of strength training on sleep apnea severity in the elderly: study protocol for a randomized controlled trial
}

\author{
Roberto Pacheco da Silva1*, Denis Martinez ${ }^{1,2}$, Pedro Lopez ${ }^{3}$ and Eduardo Lusa Cadore ${ }^{3,4}$
}

\begin{abstract}
Background: Obstructive sleep apnea (OSA) occurs due to sleep-induced upper airway muscle relaxation resulting in increased pharyngeal collapsibility. Clinical trials have shown a favorable effect of exercise training on OSA severity in middle-aged adults. Aging is characterized by motor-unit loss. Force training may affect the whole body muscle tone. We hypothesize that interventions increasing muscle strength might propagate to motor units at the abductor pharyngeal muscles, reducing collapsibility and, hence, sleep apnea severity in elderly patients with obstructive sleep apnea.

Methods/design: This is a randomized clinical trial including patients between 65 and 80 years of age, with obstructive sleep apnea, and an apnea-hypopnea index (AHI) between 20 and 50 events/hour, diagnosed by out-of-center in-home type III polysomnography. Forty subjects will be included and randomly assigned to two equal sized groups. The participants allocated to the intervention group will attend two sessions per week of one-hour strength training for the legs, arms, chest, back, and abdomen and the controls will receive advice on lifestyle change. The primary outcome measure of the study will be the change in apnea-hypopnea index and the secondary outcomes will be the body composition, evaluated by anthropometric and bioelectrical impedance variables; maximum dynamic force, appraised by one-repetition maximum strength test; muscle quality and thickness by ultrasound; physical function assessed by sit-to-stand test, timed up and go test, handgrip strength test. The study duration will be 12 weeks. Intention-to-treat and per-protocol analyses will be performed.

Discussion: The high prevalence of obstructive sleep apnea in elderly people is a public health issue. OSA is a recognized cause of cardiovascular disease and reduces quality of life due to sleepiness and fatigue. Exercise is a low-cost intervention that could help to detain the trend towards age-dependent loss of pharyngeal motor units and progressive severity of obstructive sleep apnea. Home-based strength exercises may represent a more practical approach than aerobic exercise for elderly patients. If the results confirm our hypothesis, further research on the clinical application of our findings will be warranted.
\end{abstract}

Trial registration: ClinicalTrials.gov, NCT02742792. Registered on 1 April 2016.

Keywords: Exercise, Training, Sleep apnea, Elderly, Strength, Functional capacity

\footnotetext{
* Correspondence: roberto.pacheco01@gmail.com

${ }^{1}$ Graduate Program in Cardiology and Cardiovascular Sciences, Universidade

Federal do Rio Grande do Sul (UFRGS), Porto Alegre, RS, Brazil

Full list of author information is available at the end of the article
} 


\section{Background}

Obstructive sleep apnea (OSA) causes repeated surges of hypoxia and awakenings during sleep [1]. OSA occurs in up to one third of the population [2], constituting a public health problem [3]. The prevalence of sleep apnea increases with age. In young women, the prevalence is $1.4 \%$. In both men and women 70 years or older, it reaches $90 \%$ and more $[2,4]$. This remarkable increase in prevalence is explainable, at least in part, by escalating body weight with age, but several contributing factors, which deserve medical attention, should be considered.

The repeated arousals caused by the need to restore breathing after each one of the recurrent breathing interruptions have as a consequence sympathetic hyperactivity, somnolence, and fatigue. Intermittent hypoxia is associated to oxidative stress, inflammation, and cardiovascular impairments [5]. Periods of sleep-dependent hypoxia also cause motor cortex dysfunction [6] Intermittent hypoxia was shown to cause demyelination [7] and neuronal damage, notably in the cerebellum [8]. In addition, OSA is a risk factor for hypertension [9], coronary artery disease [10], heart failure [11], and arrhythmias [12].

Treatment options for OSA are several and the most effective is the use of a continuous positive airway pressure (CPAP) device [13]. Oral appliances for mandibular advancement [14, 15], surgery [16], weight reduction, and lifestyle change, including recommendation of regular exercise [17] are alternatives to CPAP.

Physical exercise is accepted culturally and scientifically as a non-pharmacological intervention beneficial to health $[18,19]$. Aerobic and resistance training improves general wellbeing and particularly sleep quality [20, 21]. A sedentary lifestyle is linked with higher prevalence and severity of sleep apnea. On the other hand, exercise gives consistent results in treating OSA, and increasing the number of hours of exercise is associated with reduction in the severity of OSA $[22,23]$.

In experimental studies, the effect size of exercise in the treatment of OSA ranges between 0.6 [24] and 1.5 [25] standard deviations. In a meta-analysis of five studies, totaling 129 participants, the mean effect is a reduction of seven events per hour [26]. All randomized clinical trials and other studies have involved participants between 42 and 54 years of age on average. No study involving the most affected population, elderly individuals, was identified in extensive PubMed and Embase searches. The decrease in physical fitness in the elderly is associated with motor unit loss [27, 28], falls [29, 30], pain [31], musculoskeletal changes, and sleep disorders [32, 33]. Moreover, the worsening of overall physical function is a predictor of mortality [34].

The relevance of strength training in elderly people is linked to the medical consequences of muscle fiber loss. Sarcopenia starts at the age of 35 years and 45 years, in men and women, respectively [35-37], and progresses at a rate of $1-2 \%$ per year after the sixth decade [38]. It is aggravated by sedentary lifestyle, reduced levels of trophic hormones, and neurodegenerative processes $[39,40]$. It is plausible that weakening of the pharyngeal abductor muscles participates in the agerelated increase in the prevalence of OSA. Hypothetically, a widespread tonic effect [41] could lead to improvement in pharyngeal abductor function, bettering airway patency during sleep.

The increase in OSA prevalence with ageing may be linked also to overnight rostral fluid displacement from the legs [42]. Leg fluid volume correlates with the apnea-hypopnea index (AHI) and with the number of hours sitting, suggesting that "a sedentary way of life may predispose to OSA" [43].

Despite the abundant evidence on the effects of resistance training on the neuromuscular function, the issue of deleterious effects of OSA on muscle function in elderly subjects remains uninvestigated. Moreover, no highlevel evidence on the effects of strength training on the severity of OSA in elderly people was found.

\section{Rationale}

The systemic improvement in muscle strength after resistance training may reach the abductor pharyngeal muscle and improve OSA. Hypothetically, a widespread tonic effect would lead to an improvement in pharyngeal abductor function and bettering of airway patency during sleep. Also, reduction in sedentarism may reduce fluid retention. Reduction in the severity of OSA induced by physical training has been shown in randomized clinical trials and meta-analyses, but none of the studies to date have investigated the effect of strength training on sleep apnea severity in elderly individuals.

\section{Research question}

Will elderly people performing resistance training reduce the AHI in comparison with a control group performing recreational physical activity?

\section{Methods/design \\ Study design}

This study is a parallel randomized, blind, controlled superiority trial. The study strategy is registered, constructed and presented according to the recommendations for interventional trials [44] (see Additional file 1 for the Standard Protocol Items: Recommendations for Interventional Trials (SPIRIT) checklist). 


\section{Eligible participants}

Inclusion criteria Patients should fulfill the following criteria:

- Age between 65 and 80 years

- Either gender

- Not being engaged in regular structured resistance exercise

- AHI between 20 and 50 events per hour

- Availability of time to include physical activity in their routine

- Consenting to participate in the survey

\section{Exclusion criteria}

- Being in treatment for sleep apnea

- Osteoarticular injuries or illnesses that impair the performance of the exercises included in the project

- Neuromuscular problems

- Uncontrolled hypertension

- Acute myocardial infarction or stroke in the last year

- Recent trauma to the upper airway

- Other serious chronic disease with treatment for over a month in the last year

- Regular or abusive use of medications or drugs with effects on the central nervous system

\section{Sample size}

The sample size calculation for this trial was performed using the G-Power program (Franz Faul, Universität Kiel, Germany) [45]. We chose an effect size of 0.6 standard deviations described as the lowest among five articles included in a meta-analysis of the effect of exercise studies on sleep apnea [24] as the target for our study. For two groups (training and control) and two assessments (before and after 12 weeks of training), with $90 \%$ power and probability of alpha error of $5 \%$, the sample size calculated for analysis of variance with repeated measures would be 16 patients in each group. To compensate for possible participant loss, 40 subjects will be included. Losses after randomization will be included in the intention-to-treat but not in the perprotocol analyses.

\section{Randomization process}

Randomization will be performed using a sequence of numbers generated by computer at randomization.com. Patients will be assigned to the intervention or control groups by a researcher with no information about the participants and not otherwise involved in the protocol.

\section{Blinding}

The evaluators taking measurements for outcomes will be blinded to the group assignment. The physical educator responsible for prescribing and monitoring the training will not be blinded to the groups and will not be involved in other steps of the protocol. A certified scorer blinded to the groups will perform the scoring of the AHI. The blinding code will be broken at the end of the study or earlier by request of regulatory committees or in the case of serious adverse events in connection with the group assignment.

\section{Recruitment}

The information technology system of the university hospital will generate a list of patients aged 65 to 80 years ascribed to the institutional primary care unit. After chart review, the subjects will be invited to join in the study through telephone calls. Those willing to participate will visit the institutional research center to undergo the informed consent process and initiate the assessment.

\section{Statistical methods}

Intention-to-treat and per-protocol analyses will be performed for the primary and secondary outcomes. Patients who do not perform the 24 training sessions or who report poor adherence to the healthy lifestyle program during 2 weeks or more will be excluded from the per-protocol analysis. Missing data will be considered as missing, without data imputation.

Means and standard deviation will be used to represent data with a normal distribution and medians and quartiles will be used to describe non-normally distributed data. Natural logarithm transformation will be used before inclusion of non-normally distributed dependent variables in analyses. The significance of the differences between groups, at the baseline, will be tested by the Student $t$ test and chi-squared test, for linear and categorical variables, respectively. Generalized estimating equations (GEE) will be used to detect differences between exercise and control groups and time"group interaction. The results with $P$ values $<0.05$ for alpha error will be considered statistically significant. Statistical analyses will be performed using SPSS software (SPSS Inc., Chicago, IL, USA).

\section{Monitoring}

Monitoring of the physical activity in the intervention group will be performed by the physical educator after each session in a specific form of the electronic case report form. Patients in the control group will record in a log sheet the number of goals met during each week. The institutional ethics committee will be 
monitoring the data. All analyses will be performed at the end of the study.

\section{Study measurements Description of intervention, comparison group, and follow up}

The patients will be allocated to strength training or lifestyle advice groups. A detailed description of the phases of the study are shown in Figs. 1 and 2.

The program will consist of a twice-a-week routine of strength training sessions over 12 weeks. The sessions will be separated by at least 48 -hour intervals. Each training session will consist of exercises for the legs, arms, chest, back, and abdomen. The subjects will be instructed to perform the concentric and eccentric phases of each repetition with controlled speed (2-3 seconds at each stage). The training will be divided into cycles of 4 weeks each with intensity and progressive exercise volumes.

- First cycle: two sets of 12-14 repetitions in the first week using $50 \%$ maximal load in order to improve the execution and motor control of exercises, followed by 3 weeks in which individuals will perform three sets of 12-14 repetitions maximum (i.e., performance of sets until concentric failure).

- Second cycle: three sets of 10-12 repetitions maximum.

- Third cycle: three sets of 8-10 repetitions maximum.
The rest interval between sets will be 1 to 1.5 minute $[46,47]$. The load for each exercise will be adjusted according to the maximal performance of each individual.

The individuals allocated to the control group will receive advice on lifestyle. In addition, individuals will be encouraged to participate in the meetings of an elderlydirected activity group in the primary care unit of the university hospital.

\section{Ethics}

When the subject enters the study, the procedures performed in the physical tests, bio impedance, polysomnography, and training will be explained in detail. The anticipated risks to participants, beyond the occasional pain due to the training effort, will be fatigue and/or dizziness during and/or at the end of exercises. All these signs and symptoms are expected and reversed after cessation of effort. Post-delayed muscle pain, lasting up to 48 hours after the exercise session is a normal effect of strength training, mainly after the first sessions of training. The patient can leave the trial at any time.

The subjects identified with AHI $<20$ or AHI $>50$, who are excluded from the study, will be submitted for consultation and treatment in the sleep research clinic in the primary care unit. At the end of the study, all volunteers will be forwarded to the sleep research clinic in the primary care unit for medical treatment.

\begin{tabular}{|c|c|c|c|}
\hline & \multicolumn{3}{|c|}{ STUDY PERIOD } \\
\hline & Enrolment & Allocation & Post-allocation \\
\hline TIMEPOINT & $-t_{1}$ & 0 & 3 months post-randomization \\
\hline ENROLMENT: & & & \\
\hline Eligibility screen & $X$ & & \\
\hline Informed consent & $\mathrm{x}$ & & \\
\hline $\begin{array}{r}\text { Baseline } \\
\text { evaluations }\end{array}$ & $\mathrm{X}$ & & \\
\hline $\begin{array}{l}\text { Randomization } \\
\text { and Allocation }\end{array}$ & & $\mathrm{X}$ & \\
\hline INTERVENTIONS: & & & \\
\hline Strength training & & & - \\
\hline Control & & & \\
\hline ASSESSMENTS: & & & \\
\hline Primary outcome & $x$ & & $\mathrm{X}$ \\
\hline $\begin{array}{r}\text { Secondary } \\
\text { outcomes }\end{array}$ & $X$ & & $X$ \\
\hline
\end{tabular}

Fig. 1 Standard Protocol Items: Recommendations for Interventional Trials (SPIRIT) figure 


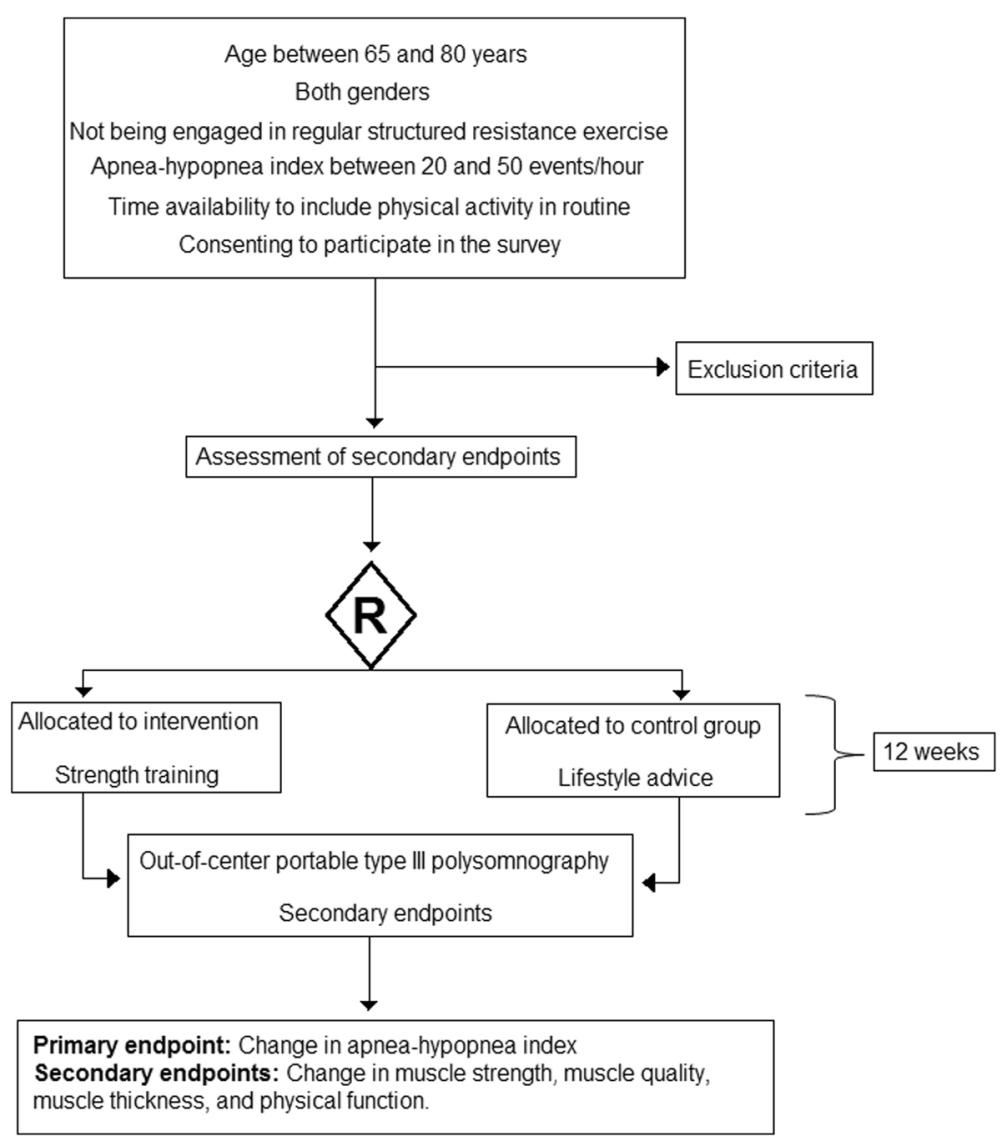

Fig. 2 Flow diagram of the study design

The trial results will be published in an international journal with impact. The investigators will communicate the trial results to participants via email. No publication restrictions will be implemented.

\section{Outcomes}

\section{Primary outcomes}

- Apnea-hypopnea index (number of apnea and hypopnea events per hour of artifact-free recording in the polygraphy)

\section{Secondary outcomes}

- Muscle strength

- Muscle thickness

- Muscle quality

- Body composition (fluid, fat)

- Physical function

\section{Follow up and duration of the study}

There will be four visits at the clinical research center for outcome evaluation. Two will be at baseline (a visit to receive the polygraph device and another visit for carrying out evaluation of body composition, physical function, and muscle strength, function, and quality) and two visits at the final follow up (reassessment of the same parameters). The study protocol consists of 12 weeks of strength training twice a week (24 sessions).

\section{Assessment of outcomes Apnea-hypopnea index}

Out-of-center polygraphy will assess the presence and severity of sleep apnea. Portable type III polysomnography monitors (Embletta Gold III, Embla, Broomfield, CO, USA; or SomnoCheck Effort, Weinmann, Hamburg, Germany) will be employed to evaluate: (1) chest effort, (2) abdominal effort, (3) oximetry, (4) nasal airflow, and (5) position, as previously described [48]. Apnea and hypopnea events, respiratory effort related arousals (RERAs), and the apnea-hypopnea and respiratory disturbance index will be reported according to the rules of the American Academy of Sleep Medicine (AASM) [49]. The AHI will define the diagnosis of sleep apnea. Polysomnography will be performed before and after 12 weeks using the same polygraph monitor on both occasions. 


\section{Muscle strength}

Subjects will perform one-repetition maximum (1-RM) tests of knee extension (unilateral) and preacher curl elbow flexion (bilateral) (Können Gym, Porto Alegre, Brazil). The same investigator, with identical subject/ equipment positioning, will conduct the pre-tests and post-tests. Before the 1-RM tests, subjects will be familiarized with the testing procedures and will perform 10 repetitions with light resistance as warm up. Thereafter, resistance will be increased until the subjects became unable to lift the additional weight using the proper technique. The time spent in each muscle action will be controlled $(\sim 2$ seconds in both concentric and eccentric phases). All 1-RM values will be determined within 3-5 attempts, with 3 min rest between each attempt. At post-testing, 1-RM will be performed 3-5 days after the last training session.

\section{Muscle thickness}

Quadriceps femoris B-mode ultrasound images will be obtained with a $38-\mathrm{mm}, 9.0 \mathrm{MHz}$ linear-array probe (image $70 \mathrm{~mm}$ depth; $90 \mathrm{~dB}$ general gain, time gain compensation (TGC) at a neutral position) with a Nemio XG ultrasound device (Toshiba, Japan). Before any measurement, subjects will rest in the supine position with the lower limbs extended and relaxed for $15 \mathrm{~min}$ to allow fluid shifts to stabilize [50]. Transversal images of the right vastus lateralis $(\mathrm{VL})$, rectus femoris $(\mathrm{RF})$, vastus intermedius (VI) and vastus medialis (VM) muscles will be acquired. The probe will be coated with a watersoluble transmission gel to provide acoustic contact and care will be taken to avoid the compression of the dermal surface. The measurement sites will be the same as those adopted in previous studies [51-54]. All images will be acquired and analyzed by the same trained investigator. At follow up, 3-5 days after the last training session, muscle thickness will be measured.

The computer-assisted determination of muscle thickness will use the standard function of ImageJ 1.42q software (National Institute of Health, USA). The calculation will include the distance between adipose tissuemuscle interface for VL, RF, and VM, and bone-muscle interface for VI. Whole quadriceps muscle thickness will be obtained as the sum of the four individual quadriceps portions:

$$
\begin{aligned}
\mathrm{QMT}= & (\mathrm{VL} \text { muscle thickness }+\mathrm{RF} \text { muscle thickness } \\
& +\mathrm{VI} \text { muscle thickness }+\mathrm{VM} \text { muscle thickness })
\end{aligned}
$$

\section{Muscle quality}

\section{Echo intensity}

Echo intensity (EI) will be determined according to previous studies $[52,53]$ by computer-assisted grayscale analyses using the standard function of Image $1.42 \mathrm{q}$ software (National Institute of Health, USA). Single images of each muscle will be digitized and analyzed. Regions of interest of each quadriceps muscle portion (VL, RF, VI, and VM) will be selected, including as much muscle as possible but avoiding other tissues (such as bone and surrounding fascia) for EI calculation of each component of the quadriceps femoris. The mean EI will be determined using a standard gray-scale histogram function and expressed as a value between 0 (black) and 255 (white). At follow up, EI will be performed 3-5 days after the last training session. Thereafter, the EI will be determined from the average EI value from all quadriceps muscle portions:

$$
\begin{aligned}
\mathrm{EI}= & (\mathrm{VL} \text { echo intensity }+ \text { RF echo intensity } \\
& +\mathrm{VI} \text { echo intensity }+\mathrm{VM} \text { echo intensity }) / 4
\end{aligned}
$$

\section{Specific tension}

To obtain a value for specific tension (ST), the knee extension 1-RM value will be divided by the muscle mass unit. Thus, the ST will be determining according to previous studies [53] using o the following equation:

$$
\text { Specific Tension }=1-\mathrm{RM}(\mathrm{kg}) / \mathrm{QMT}(\mathrm{mm})
$$

\section{Physical function}

Physical function will be evaluated by a battery of three tests: (1) handgrip strength, (2) sit-to-stand ability, and (3) mobility.

\section{Maximal handgrip strength}

The maximal handgrip strength will be determined in the dominant arm using a handgrip dynamometer (Jamar Hydraulic Hand Dynamometer, Sammons Preston CO, Bolingbrook, IL, USA) [55]. The maximal strength will be measured three times at intervals of $2 \mathrm{~min}$ of rest between assessments. The maximal value obtained will be used as maximal strength [56].

\section{Sit-to-stand ability}

Sit-to-stand ability will be assessed by the sit-to-stand test $[57,58]$. The test includes five complete movements of getting up and sitting in the chair during the shortest possible time $[59,60]$.

\section{Mobility}

To evaluate mobility, the timed up and go test will be used [61-63]. The performance and time during the test reflects reaction time, muscle strength of the lower limbs, balance disorders and difficulty walking $[64,65]$. This test evaluates the execution speed in getting up 
from a chair with arms, walking ahead $3 \mathrm{~m}$, turning around, walking back, and sitting in the chair [66].

\section{Discussion}

The present study is designed to evaluate the effects of resistance training on OSA severity in elderly persons. Our hypothesis is that resistance training will reduce OSA severity while improving neuromuscular function. A previous systematic review and meta-analysis showed that exercise training reduces approximately seven events in AHI compared to control groups [24]. However, little is known about the effect of resistance training alone without aerobic training on OSA in the elderly population. Also, the efficacy of this intervention remains untested in elderly populations, possibly because of prejudices about age-related frailty. None of the studies found in an ample PubMed and Embase search mentioned any randomized controlled trials (RCTs) of treatments for OSA

Resistance training is recognized as the most effective intervention to improve the muscle mass and quality, i.e., lean muscle mass, quadriceps muscle thickness, and intramuscular adipose tissue. Also, resistance training enhances the neuromuscular function, i.e., lower limb strength, and specific tension in elderly persons [67]. To the best of our knowledge this would be the first study to investigate the heterotopic effect of resistance training. Regarding the secondary outcomes, the enhancements of performance in functional tests have seldom been described in elderly people [68]. Therefore, our RCT will provide data for analyses of the relationship between OSA and functional performance.

\section{Trial status}

At the time of manuscript submission, the enrollment of volunteers is ongoing.

\section{Additional file}

Additional file 1: SPIRIT checklist. (DOC $121 \mathrm{~kb}$ )

\section{Abbreviations}

AHI: Apnea-hypopnea index; CPAP: Continuous positive airway pressure; El: Echo intensity; OSA: Obstructive sleep apnea; RCT: Randomized controlled trial; RF: Rectus femoris; SPIRIT: Standard Protocol Items: Recommendation for Interventional Trials; ST: Specific tension; VI: Vastus intermedius; VL: Vastus lateralis; VM: Vastus medialis

\section{Acknowledgements}

The authors acknowledge the contribution of colleagues from the Interdisciplinary Sleep Research Laboratory_LIPES (Cardiology Division, HCPA, UFRGS, Porto Alegre, Brazil) for their support.

\section{Funding}

The research is being supported by grant number 15-0613 from the Fundo de Incentivo à Pesquisa (FIPE), Hospital de Clínicas de Porto Alegre, Brazil. Mr. Silva received grants from the Brazilian government through Coordenação de Aperfeiçoamento de Pessoal de Nível Superior (CAPES) reviewed internally at the Graduate Program in Cardiology.

\section{Availability of data and materials \\ Not applicable.}

\section{Authors' contributions}

RPS conceived the study, participated in the design of the study and writing of the study protocol, and prepared the final version of the manuscript. He is currently screening and instructing patients regarding the protocol. DM participated in the design of the study and writing of the study protocol, and prepared the final version of the manuscript. $\mathrm{PL}$ participated in the design of the study, and is currently responsible for performing most of the blinded measurements. ELC participated in the design of the study and critical revision of the manuscript for important intellectual content. All authors read and approved the final manuscript.

\section{Authors' information}

Not applicable.

\section{Ethics approval and consent to participate}

All participants will be asked to sign the approved informed consent form prior to participation in the study. The study was approved by the Ethics Committee of the Hospital de Clínicas de Porto Alegre under the number 15-0613. This committee is accredited by the Office of Human Research Protection as an Institutional Review Board (IRB0000921). The study has been registered under the number NCT02742792 at https://clinicaltrials.gov/ct2/ show/NCT02742792. The research will be conducted according to the guidelines and regulatory standards for research involving human beings, Resolution No. 466/2012 of the National Health Council.

\section{Consent for publication}

Not applicable.

\section{Competing interests}

The authors declare that they have no competing interests.

\section{Publisher's Note}

Springer Nature remains neutral with regard to jurisdictional claims in published maps and institutional affiliations.

\section{Author details}

${ }^{1}$ Graduate Program in Cardiology and Cardiovascular Sciences, Universidade Federal do Rio Grande do Sul (UFRGS), Porto Alegre, RS, Brazil. ${ }^{2}$ Cardiology Unit, Hospital de Clinicas de Porto Alegre (HCPA), UFRGS, Porto Alegre, RS, Brazil. ${ }^{3}$ Exercise Research Laboratory, UFRGS, Porto Alegre, RS, Brazil. ${ }^{4}$ Physical Education School, UFRGS, Porto Alegre, RS, Brazil.

Received: 22 November 2016 Accepted: 5 October 2017

Published online: 23 October 2017

\section{References}

1. American Academy of Sleep Medicine. International classification of sleep disorders. 3rd ed. Darien, IL: American Academy of Sleep Medicine. 2014.

2. Tufik S, Santos-Silva R, Taddei JA, Bittencourt LR. Obstructive sleep apnea syndrome in the Sao Paulo Epidemiologic Sleep Study. Sleep Med. 2010;11(5):441-6.

3. Phillipson EA. Sleep apnea - a major public health problem. N Engl J Med. 1993:328(17):1271-3.

4. Senaratna CV, Perret JL, Lodge CJ, Lowe AJ, Campbell BE, Matheson MC, et al. Prevalence of obstructive sleep apnea in the general population: a systematic review. Sleep Med Rev. 2017;34:70-81.

5. Bradley TD, Floras JS. Obstructive sleep apnoea and its cardiovascular consequences. Lancet. 2009;373(9657):82-93.

6. Alexandre F, Heraud N, Sanchez AM, Tremey E, Oliver N, Guerin P, et al. Brain damage and motor cortex impairment in chronic obstructive pulmonary disease: implication of nonrapid eye movement sleep desaturation. Sleep. 2016:39(2):327-35.

7. Kim LJ, Martinez D, Fiori CZ, Baronio D, Kretzmann NA, Barros HM Hypomyelination, memory impairment, and blood-brain barrier permeability in a model of sleep apnea. Brain Res. 2015;1597:28-36. 
8. Baronio D, Martinez D, Fiori CZ, Bambini-Junior V, Forgiarini LF, Pase da Rosa $D$, et al. Altered aquaporins in the brains of mice submitted to intermittent hypoxia model of sleep apnea. Respir Physiol Neurobiol. 2013;185(2):217-21.

9. Peppard PE, Young T, Palta M, Skatrud J. Prospective study of the association between sleep-disordered breathing and hypertension. N Engl J Med. 2000;342(19):1378-84

10. Martinez D, Klein C, Rahmeier L, da Silva RP, Fiori CZ, Cassol CM, et al. Sleep apnea is a stronger predictor for coronary heart disease than traditional risk factors. Sleep Breath. 2012;16(3):695-701.

11. Kourouklis SP, Vagiakis E, Paraskevaidis IA, Farmakis D, Kostikas K, Parissis JT, et al. Effective sleep apnoea treatment improves cardiac function in patients with chronic heart failure. Int J Cardiol. 2012;168(1):157-62.

12. Qureshi WT, Nasir UB, Alqalyoobi S, O'Neal WT, Mawri S, Sabbagh S, et al. Meta-analysis of continuous positive airway pressure as a therapy of atrial fibrillation in obstructive sleep apnea. Am J Cardiol. 2015;116(11):1767-73.

13. Marin JM, Carrizo SJ, Vicente E, Agusti AG. Long-term cardiovascular outcomes in men with obstructive sleep apnoea-hypopnoea with or without treatment with continuous positive airway pressure: an observational study. Lancet. 2005;365(9464):1046-53.

14. Lim J, Lasserson TJ, Fleetham J, Wright J. Oral appliances for obstructive sleep apnoea. Cochrane Database Syst Rev. 2006;(1):CD004435.

15. Phillips CL, Grunstein RR, Darendeliler MA, Mihailidou AS, Srinivasan VK, Yee $B J$, et al. Health outcomes of continuous positive airway pressure versus oral appliance treatment for obstructive sleep apnea: a randomized controlled trial. Am J Respir Crit Care Med. 2013;187(8):879-87.

16. Greenburg DL, Lettieri CJ, Eliasson AH. Effects of surgical weight loss on measures of obstructive sleep apnea: a meta-analysis. Am J Med. 2009;122(6):535-42.

17. Araghi MH, Chen YF, Jagielski A, Choudhury S, Banerjee D, Hussain S, et al. Effectiveness of lifestyle interventions on obstructive sleep apnea (OSA): systematic review and meta-analysis. Sleep. 2013;36(10):1553-62. 1562A-1562E.

18. Fiuza-Luces C, Garatachea N, Berger NA, Lucia A. Exercise is the real polypill. Physiology (Bethesda). 2013;28(5):330-58.

19. Pareja-Galeano H, Garatachea N, Lucia A. Exercise as a polypill for chronic diseases. Prog Mol Biol Transl Sci. 2015;135:497-526.

20. Buman MP, King AC. Exercise as a treatment to enhance sleep. Am J Lifestyle Med. 2010;4(6):500-14.

21. Youngstedt SD. Effects of exercise on sleep. Clin Sports Med. 2005:24(2):355-65. xi.

22. Peppard PE, Young T. Exercise and sleep-disordered breathing: an association independent of body habitus. Sleep. 2004;27(3):480-4.

23. Awad KM, Malhotra A, Barnet JH, Quan SF, Peppard PE. Exercise is associated with a reduced incidence of sleep-disordered breathing. Am J Med. 2012;125(5):485-90

24. Servantes DM, Pelcerman A, Salvetti XM, Salles AF, de Albuquerque PF, de Salles FC, et al. Effects of home-based exercise training for patients with chronic heart failure and sleep apnoea: a randomized comparison of two different programmes. Clin Rehabil. 2012;26(1):45-57.

25. Kline CE, Ewing GB, Burch JB, Blair SN, Durstine JL, Davis JM, et al. Exercise training improves selected aspects of daytime functioning in adults with obstructive sleep apnea. J Clin Sleep Med. 2012;8(4):357-65.

26. Iftikhar $\mathrm{H}$, Kline CE, Youngstedt SD. Effects of exercise training on sleep apnea: a meta-analysis. Lung. 2014;192(1):175-84

27. Pišot R, Marusic U, Biolo G, Mazzucco S, Lazzer S, Grassi B, et al. Greater loss in muscle mass and function but smaller metabolic alterations in older compared with younger men following 2 wk of bed rest and recovery. J Appl Physiol (1985). 2016;120(8):922-9.

28. English $\mathrm{KL}$, Paddon-Jones D. Protecting muscle mass and function in older adults during bed rest. Curr Opin Clin Nutr Metab Care. 2010;13(1):34-9.

29. Kuo HK, Yang CC, Yu YH, et al. Gender specific association between selfreported sleep duration and falls in high functioning older adults. J Geronto I A Biol Sci Med Sci. 2010;65A:190-6.

30. Latimer Hill E, Cumming RG, Lewis $R$, et al. Sleep disturbances and falls in older people. J Gerontol A Biol Sci Med Sci. 2007;62A:62-6.

31. Bindawas SM, Vennu V. Longitudinal effects of physical inactivity and obesity on gait speed in older adults with frequent knee pain: data from the Osteoarthritis Initiative. Int J Environ Res Public Health. 2015;12(2):1849-63.

32. Stone KL, Blackwell TL, Ancoli-Israel S, Cauley JA, Redline S, Marshall LM. Osteoporotic Fractures in Men Study Group, et al. Sleep disturbances and risk of falls in older community-dwelling men: the outcomes of sleep disorders in older men (MrOS Sleep) Study. J Am Geriatr Soc. 2014;62(2):299-305.
33. Wilkin LD, Haddock BL. Health-related variables and functional fitness among older adults. Int J Aging Hum Dev. 2010;70(2):107-18.

34. De Buyser SL, Petrovic M, Taes YE, Toye KR, Kaufman JM, Goemaere S. Physical function measurements predict mortality in ambulatory older men. Eur J Clin Invest. 2013;43(4):379-86.

35. Doherty TJ, Vandervoort AA, Taylor AW, Brown WF. Effects of motor unit losses on strength in older men and women. J Appl Physiol. 1993;74:868-74.

36. Brown M, Hasser EM. Complexity of age-related change in skeletal muscle. J Gerontol Biol Sci. 1996;51A:B117-23.

37. Kyle UG, Genton L, Slosman DO, Pichard C. Fat-free and fat mass percentiles in 5225 healthy subjects aged 15 to 98 years. Nutrition. 2001;17(7-8):534-41.

38. Vandervoort AA. Aging of the human neuromuscular system. Muscle Nerve. 2002;25(1):17-25

39. Cadore EL, Lhullier FL, Pinto RS, Albertons CL, Pinto S, Tartaruga MP, et al. Correlations between serum hormones, strength and endurance in healthy elderly South-American men. J Sports Med Phys Fitness. 2013;53(3):255-60.

40. Cadore EL, Pinto RS, Bottaro M, Izquierdo M. Strength and endurance training prescription in healthy and frail elderly. Aging Dis. 2014;5(3):183-95.

41. Folland JP, Williams AG. The adaptations to strength training: morphological and neurological contributions to increased strength. Sports Med. 2007;37(2):145-68.

42. Yumino D, Redolfi S, Ruttanaumpawan P, Su MC, Smith S, Newton GE, et al. Nocturnal rostral fluid shift: a unifying concept for the pathogenesis of obstructive and central sleep apnea in men with heart failure. Circulation. 2010;121(14):1598-605.

43. Redolfi S, Yumino D, Ruttanaumpawan P, Yau B, Su MC, Lam J, et al. Relationship between overnight rostral fluid shift and obstructive sleep apnea in nonobese men. Am J Respir Crit Care Med. 2009;179(3):241-6.

44. Chan AW, Tetzlaff JM, Altman DG, Dickersin KDM. SPIRIT 2013: new guidance for content of clinical trial protocols. Lancet. 2013;381:91-2.

45. Faul F, Erdfelder E, Lang AG, Buchner A. G*Power 3: a flexible statistical power analysis program for the social, behavioral, and biomedical sciences. Behav Res Methods. 2007;39(2):175-91.

46. Farah BQ, Lima AH, Lins-Filho OL, Souza DJ, Silva GQ, Robertson RJ, et al. Effects of rest interval length on rating of perceived exertion during a multiple-set resistance exercise. Percept Mot Skills. 2012;115(1):273-82.

47. de Salles BF, Simão R, Miranda F, Novaes Jda S, Lemos A, Willardson JM. Rest interval between sets in strength training. Sports Med. 2009;39(9):765-77.

48. De Oliveira ACT, Martinez D, Vasconcelos LF, Goncalves SC, Lenz MC, Fuchs SC, et al. Diagnosis of obstructive sleep apnea syndrome and its outcomes with home portable monitoring. Chest. 2009;135(2):330-6.

49. Berry RB, Richard B, Gottlieb DJ, et al. The AASM manual for the scoring of sleep and associated events, Rules, terminology and technical specifications. Darien, Illinois: American Academy of Sleep Medicine; 2012.

50. Berg HE, Tedner B, Tesch PA. Changes in lower limb muscle cross-sectional area and tissue fluid volume after transition from standing to supine. Acta Physiol Scand. 1993;148:379-85.

51. Wilhelm EN, Rech A, Minozzo F, Radaelli R, Botton CE, Pinto RS. Relationship between quadriceps femoris echo intensity, muscle power, and functional capacity of older men. Age (Dordr). 2014;36:9625.

52. Rech A, Radaelli R, Goltz FR, da Rosa LH, Schneider CD, Pinto RS. Echo intensity is negatively associated with functional capacity in older women. Age (Dordr). 2014;36:9708

53. Korhonen MT, Mero AA, Alén M, Sipilä S, Häkkinen K, Liikavainio T, et al. Biomechanical and skeletal muscle determinants of maximum running speed with aging. Med Sci Sports Exerc. 2009;41:844-56.

54. Pinto RS, Correa CS, Radaelli R, Cadore EL, Brown LE, Bottaro M. Short-term strength training improves muscle quality and functional capacity of elderly women. Age (Dordr). 2014;36:365-72.

55. Innes E. Handgrip strength testing: a review of the literature. Aust Occup Ther J. 1999:46:120-40

56. Roberts HC, Denison HJ, Martin HJ, Patel HP, Syddall H, Cooper C, et al. A review of the measurement of grip strength in clinical and epidemiological studies: towards a standardised approach. Age Ageing. 2011:40:423-9.

57. Csuka M, McCarty DJ. Simple method for measurement of lower extremity muscle strength. Am J Med. 1985;78(1):77-81.

58. Bohannon RW. Sit-to-stand test for measuring performance of lower extremity muscles. Percept Mot Skills. 1995;80:163-6.

59. Guralnik JM, Ferrucci L, Simonsick EM, Salive ME, Wallace RB. Lowerextremity function in persons over the age of 70 years as a predictor of subsequent disability. N Engl J Med. 1995;332(9):556-61. 
60. Whitney SL, Wrisley DM, Marchetti GF, Gee MA, Redfern MS, Furman JM. Clinical measurement of sit-to-stand performance in people with balance disorders: validity of data for the Five-Times-Sit-to-Stand Test. Phys Ther. 2005:85(10):1034-45.

61. Bohannon RW, Schaubert K. Long-term reliability of the timed up-and-go test among community-dwelling elders. J Phys Ther Sci. 2005;17(2):93-6.

62. Thrane G, Joakimsen RM, Thornquist E. The association between timed up and go test and history of falls: the Tromso study. BMC Geriatr. 2007;7(1):1-7.

63. Beauchet O, Annweiler C, Assal F, Brindenbaugh S, Herrmann FR, Kressig RW, et al. Imagined Time Up \& Go test: a new tool to assess higher-level gait and balance disorders in older adults? J Neurol Sci. 2010;294:102-6.

64. Lamoureux E, Sparrow WA, Murphy A, Newton RU. The effects of improved strength on obstacle negotiation in community-living older adults. Gait Posture. 2003;17(3):273-83.

65. Steffen TM, Hacker TA, Mollinger L. Age-and gender-related test performance in community-dwelling elderly people: Six-Minute Walk Test, Berg Balance Scale, Timed Up \& Go Test, and gait speeds. Phys Ther. 2002;82(2):128-37.

66. Podsiadlo D, Richardson S. The timed "Up \& Go": a test of basic functional mobility for frail elderly persons. J Am Geriatr Soc. 1991;39:142-8.

67. Izquierdo M, Cadore EL. Muscle power training in the institutionalized frail: a new approach to counteracting functional declines and very late-life disability. Curr Med Res Opin. 2014;30(7):1385-90.

68. Cadore EL, Casas-Herrero A, Zambom-Ferraresi F, Idoate F, Millor N, Gómez $M$, et al. Multicomponent exercises including muscle power training enhance muscle mass, power output, and functional outcomes in institutionalized frail nonagenarians. Age (Dordr). 2014;36(2):773-85.

\section{Submit your next manuscript to BioMed Central and we will help you at every step:}

- We accept pre-submission inquiries

- Our selector tool helps you to find the most relevant journal

- We provide round the clock customer support

- Convenient online submission

- Thorough peer review

- Inclusion in PubMed and all major indexing services

- Maximum visibility for your research

Submit your manuscript at www.biomedcentral.com/submit
Biomed Central 